\title{
Bearing capacity of elements of a gas transportation system
}

\author{
Narine Pirumyan* and Mihran Stakyan \\ National University of Architecture and Construction of Armenia, Yerevan, Teryan 105, \\ Armenia
}

\begin{abstract}
The issues of improving the methods of calculation and design of the gas transmission system (GTS) are considered, taking into account the analysis of loading modes and peculiarities of its individual nodes. The analysis of design schemes for evaluating the performance of elements of the gas transmission system according to the strength criterion is given, and a probabilistic method for assessing the reliability of butt welds of pipelines is selected. The features of the operating conditions of the system are considered and the method for calculating equivalent strength indicators and loading modes is presented. A unified approach is applied for determining the probability level of failure-free operation of parts and components of a structure. A comparative analysis of traditional and new methods for assessing the performance of the considered system is given. The interval values of the parameters included in the dependence to determine the probability level of a failure-free operation of a welded joint are clarified. A computational method is proposed, which allows to increase the carrying capacity of the GTS in the probabilistic aspect.
\end{abstract}

\section{Introduction}

The efficiency and reliability of elements of building structures are the main conditions for the efficient operation of the gas transmission system (GTS). At the same time, strength is the main criterion of working capacity, and ensuring the strength reliability of structural elements is the main task of designing these systems [1-4].

Traditional methods of strength calculations are usually performed according to three calculation schemes [3.5]:

$$
\sigma=F / A \leq[\sigma], \quad A \geq F /[\sigma], \quad F \leq A[\sigma],
$$

where $[\sigma]=\sigma_{R} / s$; F - power factor; A - geometrical parameter of the structure; $\sigma, \sigma_{R},[\sigma]$ _ calculated, marginal and permissible values of stresses; s - is the safety factor.

$\sigma_{R}$ values are selected according to standards and reference data or are determined experimentally. However, calculations (1) for permissible stresses have certain drawbacks, since (1) does not represent the ratio of active and ultimate stresses, and the value $\left[{ }^{\sigma}\right]$ is

\footnotetext{
*Corresponding author: science@nuaca.am
} 
conditional, not reflecting loading conditions and not taking into account numerous factors affecting the strength of the structure [6 ]. Under these conditions, some accuracy is introduced by the calculation of the permissible safety factor [s]

$$
\boldsymbol{s}=\sigma_{R} / \sigma_{\max } \geq[s]
$$

where $\sigma_{\max }$ is the maximum effective voltage.

To ensure the required accuracy of calculations, a differential method is used to determine $[\boldsymbol{S}$ ]

$[s]=\left[s_{1}\right] \cdot\left[s_{2}\right] \cdot\left[s_{3}\right]$

which considers: [ ${ }^{\boldsymbol{s}_{1}}$ ] $=1.05 \ldots 1.20$ - the quality and homogeneity of the microstructure of the material;

$\left[\boldsymbol{S}_{2}\right]=1.1 \ldots 1.3$ - working conditions and the degree of responsibility of the structural element; $\left[{ }^{\boldsymbol{S}_{3}}\right]=1.2 \ldots 1.3$ - the accuracy of the adopted indicators of strength and design schemes. As a result: $[\boldsymbol{S}]=1.39 \ldots 2.03$, which is lower than the generally accepted interval: ([ $\boldsymbol{S}]=1,5 \ldots .3,0)$ and provides some reduction in the size and material intensity of the structure [7].

\section{Methods}

In the expressions (1), (2), all parameters are treated as deterministic and their median values are used in calculations. But all of them are random variables, basically obeying the normal or logarithmically normal distribution law. Similarly, the real modes of loading of structures in most are random, caused by the stochastic nature of the flow of production and operational processes [8-10].

As shown by computational practice [3], the use of expressions (1), (2) with median values of their parameters may lead to an increase in the material intensity of structures by $15 \ldots$ $20 \%$, which is unacceptable in up-to-date conditions, characteristic of the development of large engineering structures and main GTS, as well as in mass output of products and equipment for various purposes. This is also dictated by the requirements of expanding construction projects and infrastructure, as well as increasing the capacity, productivity and operating speeds of manufactured products and equipment [11,12].

In order to fully utilize the entire life of the bearing capacity of a GTS structure with minimum material consumption, it is advisable to switch to probability models for reliability by the strength criterion instead of the design schemes (1), (2), which ensure the condition $[6,13]$ pass to the probabilistic methods of calculating the reliability of strength criterion allowing for the specified design service life for the required level of probability of trouble-free operation to ensure the condition of $[6,13]$

$$
\left(\sigma_{\max }-\sigma_{R}\right)<0 \quad \text { or } \quad z_{p} S=\bar{\sigma}_{\max }-\bar{\sigma}_{R},
$$

where $\bar{\sigma}_{\max }, \bar{\sigma}_{R}$ - the median values of the maximum and maximum stress of the pipeline material; $S=\sqrt{S_{\sigma_{\max }}^{2}+S_{\sigma_{R}}^{2}}$-SD differences $\left(\bar{\sigma}_{\max }-\bar{\sigma}_{R}\right) ; S_{\sigma_{\max }}^{2}, S_{\sigma_{R}}^{2}$ - the same, for $\bar{\sigma}_{\max }$ and $\bar{\sigma}_{R} ; z_{p}$. the quantile of the normalized normal distribution function 
$\Phi(z)=(1 / \sqrt{2 \pi}) \int_{-\infty}^{0} \exp (-z) d z$ , corresponding to the accepted probability level of failure-free operation $\Phi(z)$ (usually taken $=0.950 \ldots 0.999$ ). Entering in (3) the coefficients of variation $v_{\sigma_{\max }}=S_{\sigma_{\max }} / \bar{\sigma}_{\max }, v_{\sigma_{R}}=S_{\sigma_{R}} / \bar{\sigma}_{R}$, and also $\overline{\mathbf{s}}=\bar{\sigma}_{R} / \bar{\sigma}_{\text {max }}$, after transformations, we obtain an expression that combines the design schemes (1) - (3) and allows us to make an assessment of the reliability of the main factors acting on the structural strength of the GTS [6,14]:

$$
z_{p}=-(\overline{\mathbf{s}}-1) \bar{\sigma}_{R} / \sqrt{\left(\overline{\mathbf{s}} v_{\sigma R}\right)^{2}+v_{\sigma \max }^{2}} .
$$

For the reasonable application of the relation (4) it is necessary to take into account all the factors of constructive, technological and operational origin, affecting the strength reliability of the GTS. But in the main pipelines of the GTS, basically, butt welds are used (fig. 1a), which of all other types are the most advanced and technological. But defects and technological imperfections of seams can significantly reduce their carrying capacity and reliability. Most often, the following types of defects are encountered (fig. 1b): 1 undercuts that change the gap in the joint along the entire perimeter and weaken the working section of the weld elements in the transitional and most stressful area of $\sigma_{2 \max }$ operation (fig. 1c): 2 - gas pores and non-metallic inclusions, that break the uniformity and become microvolumes for the occurrence and development of fatigue cracks; 3 - lack of penetration in the lower zone of the seam, weakening the bond between the deposited and base metal [5].

To the specified factors of a constructive and technological nature, an operational one is also added, which, due to the non-stationary mode of operation of the GTS, considers the specification of its operating parameters. Strength calculation of pipelines has distinctive features - a complex stress state arises in the pipe walls, and the network loading mode is mostly non-stationary and often stochastic. This implies in the calculations to apply a twostage transition to equivalent parameters: $\mathrm{a}$ - from a complex to a linear stress state; $\mathrm{b}$ from non-stationary loading mode to equivalently stationary.

\section{Results}

\section{(Method of determining equivalent parameters)}

For pipelines, in the first approximation, the design case of loading a thin-walled vessel with internal pressure $\mathrm{p}$ occurs, in which walls a flat stress $\sigma_{1}>\sigma_{2}$ occurs (element A, fig. 1a), and on the circumferential weld perpendicular stress $\sigma_{2}$ acts (section BB of element A, fig. 1a). 


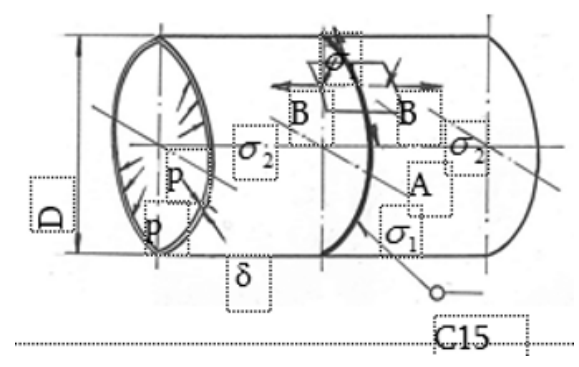

$a$

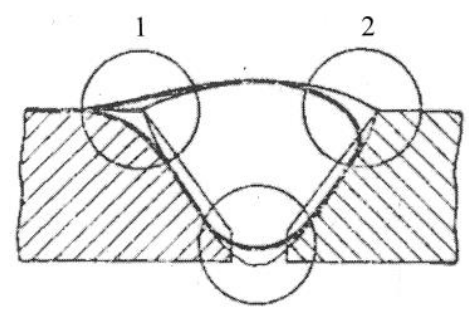

3

b

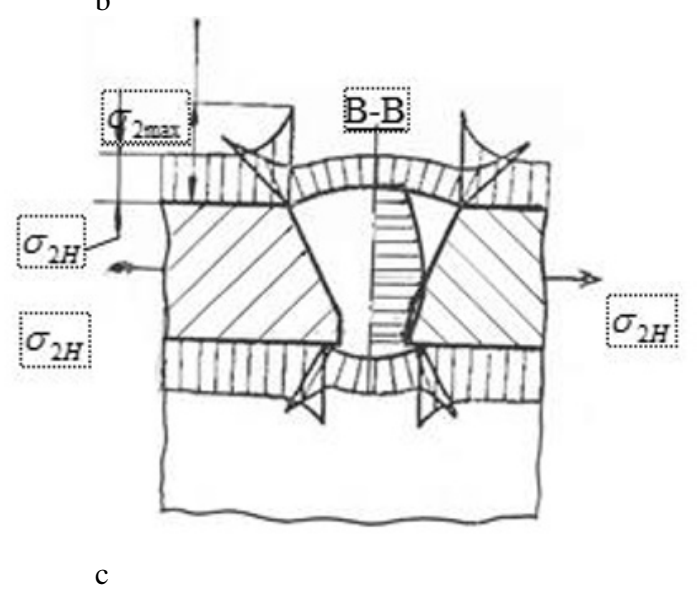

Fig. 1. Stress distribution in the weld zone: a - joint of the weld; $b$ - distribution of defects in the seam; c - diagrams of stresses in the cross section of the seam

According to [5]

$$
\sigma_{1}=p D / 2 \delta, \sigma_{2}=p D / 4 \delta, \sigma_{3}=0
$$

and the equivalent stress at $v \approx 1$ (for low carbon structural steels) and the energy theory of strength usage:

$$
\sigma_{\ni}=\sqrt{\frac{1}{2}\left(\sigma_{1}-\sigma_{2}\right)^{2}+\sigma_{1}^{2}+\sigma_{2}^{2}}=p D / 2,31 \delta .
$$

In a welded seam it can be written that, $\bar{\sigma}_{2 \max }=\sigma_{2 H} K_{\sigma u}$, where $K_{\sigma u}=1.2 \ldots 1.4$ is the stress concentration coefficient for butt joints, and $\sigma_{2 H}$ is the nominal voltage that is accepted $\bar{\sigma}_{2 H}=\sigma_{\ni}$. 
In the calculations of the strength mode loading is represented by the maximum pressure $p_{\max }$ in the pipeline, which is then at the presence of non-stationary loading is replaced by the equivalent value pmaxэ. As shown in [15],

the pressure $\mathrm{p}$ in the gas pipeline may vary within $p_{\min } \leq p \leq p_{\max }$, and in emergency situations - $0 \leq p_{i} \leq p_{\max }$, which implies a clarification of the $\sigma_{2 H}$ magnitude, and hence $\sigma_{2 \max }$. The most common options for which $p_{i}=f(t)$ is a specific time function, or $p_{i}$ values obey the normal distribution law, the functions of which are presented in fig. 2

Calculations on the bearing capacity of structures under non-stationary loading conditions are based on the principle of linear summation of cyclic damage $[3,6]$

$$
\sum N_{i} / N_{i p}=a
$$

where $N_{i}$ is the number of loading cycles at the given $p_{i} ; N_{i p}$ - cyclic durability at the onset of failure with the same $p_{i}$, determined from the equation of the fatigue curve of the

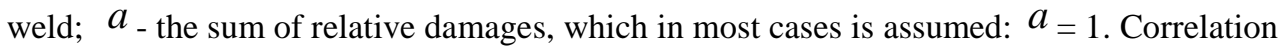
(6) allows the variable loading mode to be replaced by an equivalent stationary mode using the equivalent maximum load ( $\sigma_{2 \max \text { э }}$ stress) and $N_{\text {э }}$ cyclic durability in the calculations. Using the equation of the fatigue curve of the seam, according to (6) we can write that [1618]

$$
\begin{aligned}
& \sum p_{i}^{m} N_{i}=p_{\max \ni}^{m} N_{\max \ni p} \quad \text { or } \quad p_{\max \ni}=\sqrt[m]{\sum p_{i}^{m} N_{i} / N_{\max \ni p}}, \text { from which } \\
& p_{\max \ni}=p_{\max } \sqrt[m]{\sum\left(p_{i} / p_{\max }\right)^{m} N_{i} / N_{\max \ni p}}=K_{p} p_{\max }
\end{aligned}
$$

where $N_{\max э p}$ is the equivalent number of loads corresponding to $p_{\max \ni ;} K_{p}$ coefficient of loading mode; $\quad \mathrm{m}=4 \ldots 6$ is the degree of the equation of the fatigue curve of the welded joint; $p_{\max }$ - the maximum pressure in the pipeline, which is taken from the graph of the loading spectrum of the GTS.

This means that in a non-stationary loading mode, it is necessary to clarify the $\overline{\sigma_{2 \max \ni}}=\sigma_{2 \mu \vartheta} \alpha_{\sigma u}$ value, which takes the form

$$
\overline{\sigma_{2 \max \ni}}=\sigma_{2 \mu э} \alpha_{\sigma u} K_{p},
$$

where $\alpha_{\text {бш }}$ is the theoretical stress concentration coefficient of the weld. 


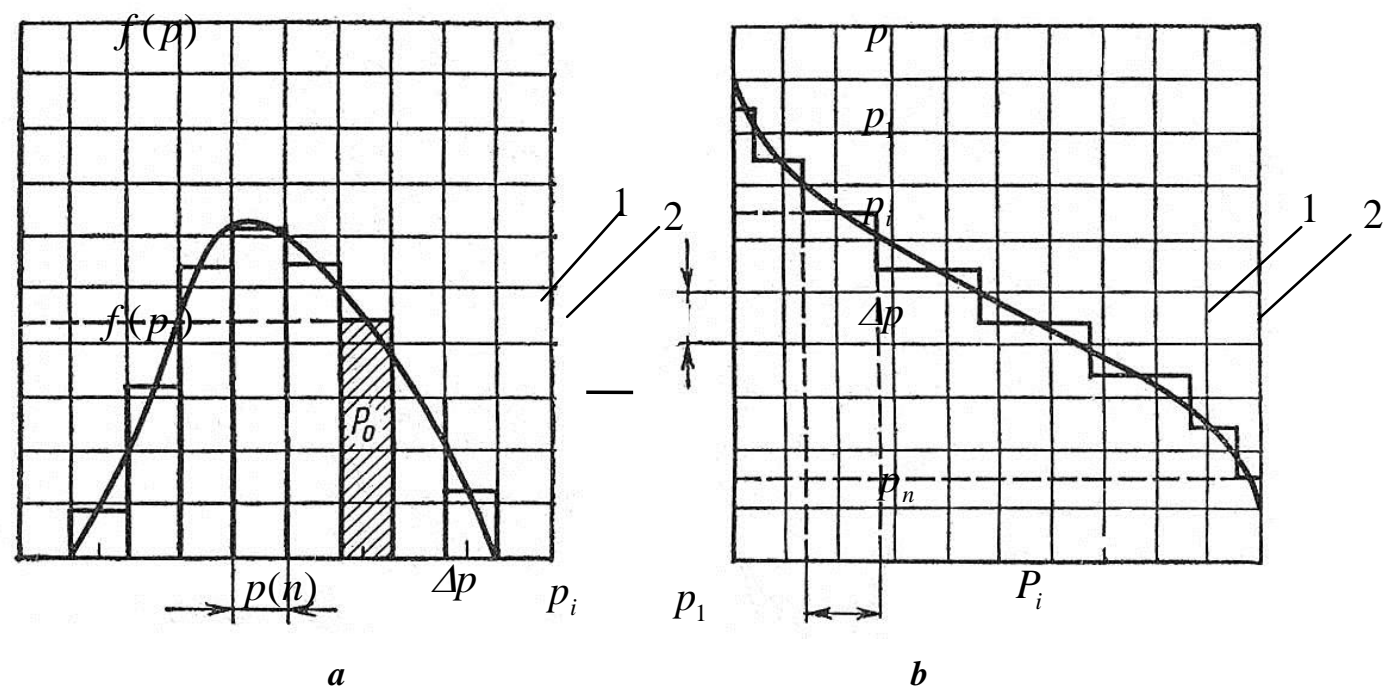

Fig. 2. Differential (a) and integral (b) distribution functions of p pressure.

Charts 1 and 2, respectively, refer to the smooth and stepwise $p_{i}$ loading conditions

Taking into account the action of the main factors, the median values $\bar{\sigma}_{R}$ are determined according to the formula

$$
\bar{\sigma}_{R}=\bar{\sigma}_{R 0} K_{\ell} K_{y} K_{c} / K_{\sigma}
$$

where $\bar{\sigma}_{R 0}$ is the endurance limit of the laboratory sample $\left(d_{0}=7,5 \ldots 10 \mathrm{~mm}\right)$; $K_{\ell}, K_{y}, K_{c}, K_{\sigma}$ - coefficients, respectively, taking into account the scale factor, weld hardening, physical and mechanical state of the weld surface and stress concentration $[3,19]$.

Using (6) and the stress fatigue curve equation, the equivalent value of the median fatigue limit is determined

$$
\sum \sigma_{i}^{m} N_{i}=\overline{\sigma_{R \ni}^{m}} N_{\ni}=\overline{\sigma_{R}^{m}} N_{G}=\text { const, } \overline{\sigma_{R \ni}}=\bar{\sigma}_{R} \sqrt[m]{N_{G} / N_{\ni}}=\overline{\sigma_{R}} K_{N}
$$

where $K_{N} \geq 1$ is the coefficient of durability; $N_{G}=(0.5 \ldots 1.0)$. 107- base number of

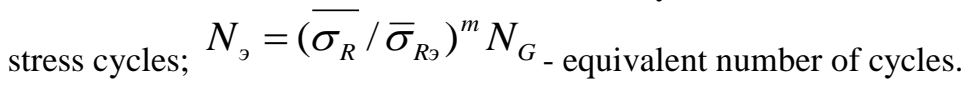

The obtained $\bar{\sigma}_{2 \max \ni \text { and }} \bar{\sigma}_{R \ni}$ values according to (8), (10) are used to clarify the median safety factor $\overline{\boldsymbol{s}}$.

\section{Discussion}

\section{(Probabilistic estimation of parameters)}

To determine the coefficients of variation $v_{\sigma R}$ and $v_{\sigma \max }$ in the first approximation, the principle of independent action of factors on the probability values of radiation is applied 


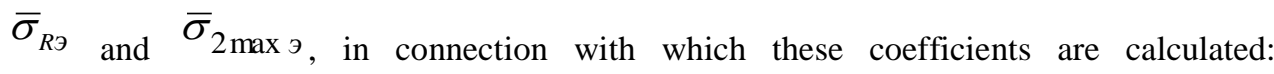
$v=\sqrt{\sum_{i=1}^{k} v_{i}^{2}}$

where $\mathrm{k}$ - the number of operational factors. Values of variation coefficients for probability values $\bar{\sigma}_{2 \max \ni \text { and }} \bar{\sigma}_{R \ni}$ must comply with typical loading conditions, as well as the characteristics of the scattering parameters of the main factors affecting the value $\bar{\sigma}_{R}$.

According to (7) and (8), the value $\overline{\sigma_{2 \max \ni}}$ is a product of three independent random variables and therefore it can be written down $[6,14]$ that

$$
v_{\sigma \max \ni}=\sqrt{v_{1}^{2}+v_{2}^{2}+v_{3}^{2}},
$$

where coefficients are introduced that take into account variations: $v_{1}=_{0,06 \ldots 0,08-}$

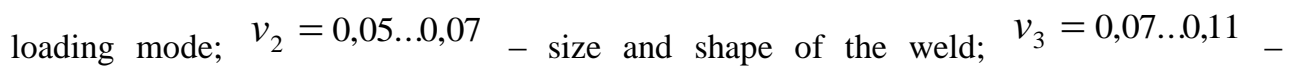
vibration $\sigma_{\max }$ in the spectrum of loading. Eventually: $v_{\sigma \max \text { э }}=0,105 \ldots 0,153$.

The value (11) for the coefficient of variation of the long endurance limit can be written down [14]:

$$
v_{\sigma R \ni}=\sqrt{v_{4}^{2}+v_{5}^{2}+v_{6}^{2}+v_{7}^{2}+v_{8}^{2}}
$$

with coefficients that take into account variations: $v_{4}=0,04 \ldots 0,06$ - the endurance limit of the material of a pipe of one heat and without a weld; $v_{5}=0,05 \ldots 0,08$ - the same, but for material from different heats; $v_{6}=0,03 \ldots 0,05$ - weld fabrication quality; $v_{7}=v_{8}=0,05 \ldots 0,06$ - respectively, the physicomechanical state of the surface layers, the geometric shape and dimensions of the seam. Eventually: $V_{\sigma R \ni}=0,092 \ldots 0,136$. Between the total values of the coefficients of variation there is a relationship $v_{\max \ni}>v_{\sigma R \ni}$, because the intervals of change of parameters of loading modes, as a rule, are wider than similar values for fatigue limits.

\section{Conclusions}

Analysis of the structure of expression (4) indicates the presence of a multiparametric dependence of quantile $z_{p}$ from the considered indicators

$$
z_{p}=F\left(\bar{s}, \bar{\sigma}_{\max \ni}, \bar{\sigma}_{R \ni}, v_{\sigma_{\vartheta} \ni}, v_{\max \ni}\right)
$$

which, in turn, depend on the parameters of various factors affecting the performance of the gas transportation system and included in the functional relationships with other parameters in the form of two- and three-parameter regression equations. This involves identifying and building a system of interrelated equations, and, on this basis - 
corresponding nomogram which allows taking into account the complex effects of all the factors and the actual operating conditions of GTS determine optimum values of the parameters and, first of all, the maximum level of probability of system uptime [20-22]. Ensuring the reliable operation of complex technical systems involves the implementation of a wide range of studies related to the design, production, testing, maintenance, diagnostics and repair of these systems. Each of these problems includes the stages of their implementation. This paper addresses the design problem of GTS, which includes the following steps:

1. The number of system elements that were previously used and tested in operation should be maximum.

2. If possible, it is necessary to apply the modular design principle more, using ready-made and tested components, mechanisms and subsystems with standard elements.

3. The system should be equipped with modern monitoring and measuring and safety devices to monitor the state of system operation, promptly identify problems and eliminate emergencies.

4. The system should be designed to ensure maximum maintainability, which allows in the shortest possible time and without interruption of the GTS to perform repair work.

This work has been carried out in the frame of "Development ant preservation of Scientificresearch sector" programme, financed by State Committee of Science of Republic of Armenia.

\section{References}

1. A.A. Ionin, Gas supply. - M. Stroiizdat, 439 (1989).

2. M.G. Sukharev, E.R. Stavrovsky, V.E. Bryansk, Optimal development of gas supply systems. - M.: Nedra, 294 (1981).

3. V.P. Kogaev, N.A., Makhutov A.P., Gusenkov, Calculations of machine parts and structures for strength and durability: Reference book. - M .: Mashinostr., 224 (1985).

4. G. B. Iosilevich, Details of machines. - M.: Mashinostr., 368 (1988).

5. I.A. Birger, B.F. Shorr, G.B. Iosilevich, Calculation on the strength of machine parts: Reference. - M.: Mashinostr., 702 (1989).

6. D.N. Reshetov, A.S. Ivanov, V.Z. Fadeev, Reliability of machines. - M.: Higher. shk., 238 (1988).

7. A.T. Skoybeda, A.V. Kuzmin, N.N. Makeychik, Machine parts and design fundamentals. - Minsk: Higher. shk., 560 (2006).

8. E.L. Volsky, A.I. Garlyauskas, V. Gerchikov, Reliability and optimal reservation of gas fields and gas pipelines. - M.: Nedra, 280 (1980).

9. V.S. Pankratov, A.V. Dubinsky, B.I. Sipershtein et al., Software and hardware of the automated process control system and experience of their operation in gas transportation associations. - Moscow: VNIIEG Gazprom, 56 (1986).

10. A.S. Pronikov, A.S. Penetrated, Parametric reliability of machines. - M.: Publishing House of Moscow State Technical University after N. E. Bauman, 560 (2002).

11. P.F. Dunayev, O.P. Lelikov, Design of units and parts of machines. - M.: Izd. Center "Academy", 496 (2008).

12. B.N. Orlov, Innovative technologies to ensure the reliability of work items and equipment. - M.: MGUPb, 328 (2013).

13. V.A. Svetlitsky, Statistical mechanics and the theory of reliability. - M.: Publishing House of Moscow State Technical University. after N.E. Bauman, 504 (2004). 
14. M.G. Stakyan, Reliability of machines and mechanisms; V. 1. - Yerevan: Publisher of the SEIU (P), 84 (2005).

15. M.G. Stakyan, A.A. Kazaryan, Yu.A. Kazaryan, Study of loading regimes of the gas transmission system. Proceedings of NUACA, N 2 (64), 120-125 (2016).

16. S.S. Chibukhchyan, M.G. Stakyan, V.V. Popov, Calculation of the reliability of components and parts of agricultural machines according to the strength criterion. International Technical and Economic Journal, N2, 64-67 (2012).

17. S.S. Chibukhchyan, M.G. Stakyan, V.V. Popov, Evaluation of the reliability of mechanical systems. Environmental Engineering, N3, 102-105(2012).

18. S.S. Chibukhchyan, M.G. Stakian, Reliability of machinery and equipment used in agriculture. Tractors and agricultural machines, N2, 43-45 (2013)

19. M.N. Stepnov, A.V. Shavrin, Statistical methods for processing the results of mechanical tests. Reference. - M.: Mashinostr., 399 (2005).

20. S.G. Akopyan, Calculation of the stationary flow distribution of the gas transmission system. Coll .: The problem of improving and developing progressive technical and economic norms and standards in the gas industry. - M.: VNIIEG Gazprom, 81-91 (1982).

21.E.A. Puchin, O.N. Didmanidze, P.P. Lezin et al., Reliability of technical systems. Moscow: Triada Medical Center, 353 (2005).

22. P.I. Orlov, Design Basics: Reference Book. 2. - M.: Mashinostr., 544 (1988). 